\title{
Clinical Practice Guidelines for the Management of Temporomandibular Joint Disorders - A Review
}

\author{
Indra Gopi ${ }^{1}$, Arvind Muthukrishnan², Maragathavalli G. ${ }^{3}$ \\ 1, 2, 3 Department of Oral Medicine and Radiology, Saveetha Dental College and Hospital, Saveetha \\ Institute of Medical and Technical Sciences (SIMATS), Kuthambakkam, Tamil Nadu, India.
}

\section{ABSTRACT}

\section{BACKGROUND}

Temporomandibular joint disorders (TMD) are a group of disorders associated with temporomandibular joints, their associated muscles, and other related structures. TMD present with pain in the joints and related structures which can radiate to the neighbouring areas mimicking ear pains, headaches, neuropathic pain and odontogenic pain. Even though TMDs are more seen in both genders, the ratio of women reporting with TMD is higher (2:1) than men. Pain, the commonest symptom encountered, is usually chronic in duration and mild to severe in intensity. Various other signs and symptoms described are clicking or popping noise, deviation of the mandible, restricted mouth opening and jaw movements. Multiple factors have been reported as an aetiology, however, there is no definite established aetiology to cause TMD. Approaches to the management of TMDs require thorough history taking, clinical and radiological assessment and proper treatment planning. The signs, symptoms, and prevalence of the diseases are also necessary considerations in the treatment planning of TMDs. Traditional approaches in the management of TMDs involve systemic medications, physical therapies, and surgical interventions. The first line of systemic medications to be advised to relieve pain are analgesics. Nonsteroidal anti-inflammatory drugs (NSAIDs) such as ibuprofen, muscle relaxants, barbiturates like benzodiazepine have been frequently administered. In recent times, extraction of impacted or buccoverted third molar teeth has also proven to be effective in reducing the pain associated with TMDs. The purpose of this study is to provide new clinical practice guidelines to establish a multidisciplinary approach in the management of patients with TMDs and to improve the patient's quality of life (QoL).

\section{KEY WORDS}

Pain, Temporomandibular Joint Disorder, TMD, Treatment Guidelines
Corresponding Author: Dr. Arvind Muthukrishnan, Professor and Head of the Department, Dept. of Oral Medicine and Radiology, Saveetha Dental College and Hospital, No. 162, Poonamallee High Rd, Velappanchavadi, Chennai - 600077, Tamil Nadu, India.

E-mail: arvindmuthukrishnan@yahoo.com

\section{DOI: $10.14260 /$ jemds $/ 2021 / 573$}

How to Cite This Article: Gopi I, Muthukrishnan A, Maragathavalli G. Clinical practice guidelines for the management of temporomandibular joint disorders - a review. J Evolution Med Dent Sci 2021;10(33):2809-2815, DOI: $10.14260 /$ jemds/2021/573

Submission 25-01-2021, Peer Review 03-05-2021, Acceptance 10-05-2021, Published 16-08-2021.

Copyright (C) 2021 Indra Gopi et al. This is an open access article distributed under Creative Commons Attribution License [Attribution 4.0 International (CC BY 4.0)] 


\section{BACKGROUND}

Temporomandibular joint is a bilaterally present, movable, synovial joint in the orofacial area which is supported by the masticatory muscles. Diseases involving the temporomandibular joint are referred to as temporomandibular joint disorders (TMD) and account for about $10-15 \%$ of adult pain. Previous studies have reported that only $5 \%$ of the TMD population seek help. ${ }^{1,2}$ Incidence of patients present with TMD age between 18 and 40 years and are more common among women. ${ }^{3}$ A survey conducted in Korea revealed that more than 750,000 outpatients visited various medical institutions in Korea for the treatment of TMD in 2015 alone. $^{4}$

The temporomandibular joint is a complex structure. To diagnose and treat the TMDs, various clinical practice guidelines (CPGs) have been introduced in the past, namely, the International Research Diagnostic Criteria for Temporomandibular Dysfunction in 2013,5,6 Diagnosis and Treatment of Temporomandibular Disorders (American Academy of Family Physicians, 2015), 7 the Guideline on Acquired Temporomandibular Disorders in Infants, Children, and Adolescents (American Academy of Paediatric Dentistry, 2015), ${ }^{8}$ and the Guidelines for Diagnosis and Management of Disorders Involving the Temporomandibular Joint and Related Musculoskeletal Structures (American Society of Temporomandibular Joint Surgeons, 2015) ${ }^{9}$ which are widely accepted CPGs that are in practice till day. The aetiology of
TMD is said to be multifaceted and it includes environmental, biological, emotional, and cognitive factors. Deleterious habits like smoking are also found to be associated with an increased risk of TMD in females younger than 30 years of age. ${ }^{10}$ Inflammatory conditions such as synovitis are often reported to cause pain at rest. Various disorders of the TMJ are classified broadly into intra-articular and extra-articular as defined by the International Research Diagnostic Criteria for Temporomandibular Dysfunction.

Treatment for TMD involves occlusal or anterior repositioning splints, cognitive behavioural therapy, and various physical therapies such as transcutaneous electrical nerve stimulation (TENS), acupuncture, massage therapy, joint mobilization, therapeutic exercises and surgical management.11-15 Population-based studies have reported that although $90-95 \%$ of the patients with TMD can be managed non-surgically, the remaining 5-10\% of the population tend to require surgical management. ${ }^{16-18}$ It is mandatory to make a precise diagnosis to ensure that the correct treatment is provided and that potentially serious problems are not ignored.

A thorough electronic literature search was carried out in PubMed Central, Google Scholar, Web of Science, LILACS and Science direct databases. The articles were thoroughly scrutinized, and only associated articles were taken into contemplation. This study intended to critically analyse all the associated literatures of TMD taken and provide new clinical practice guidelines in the management of patients with TMDs.

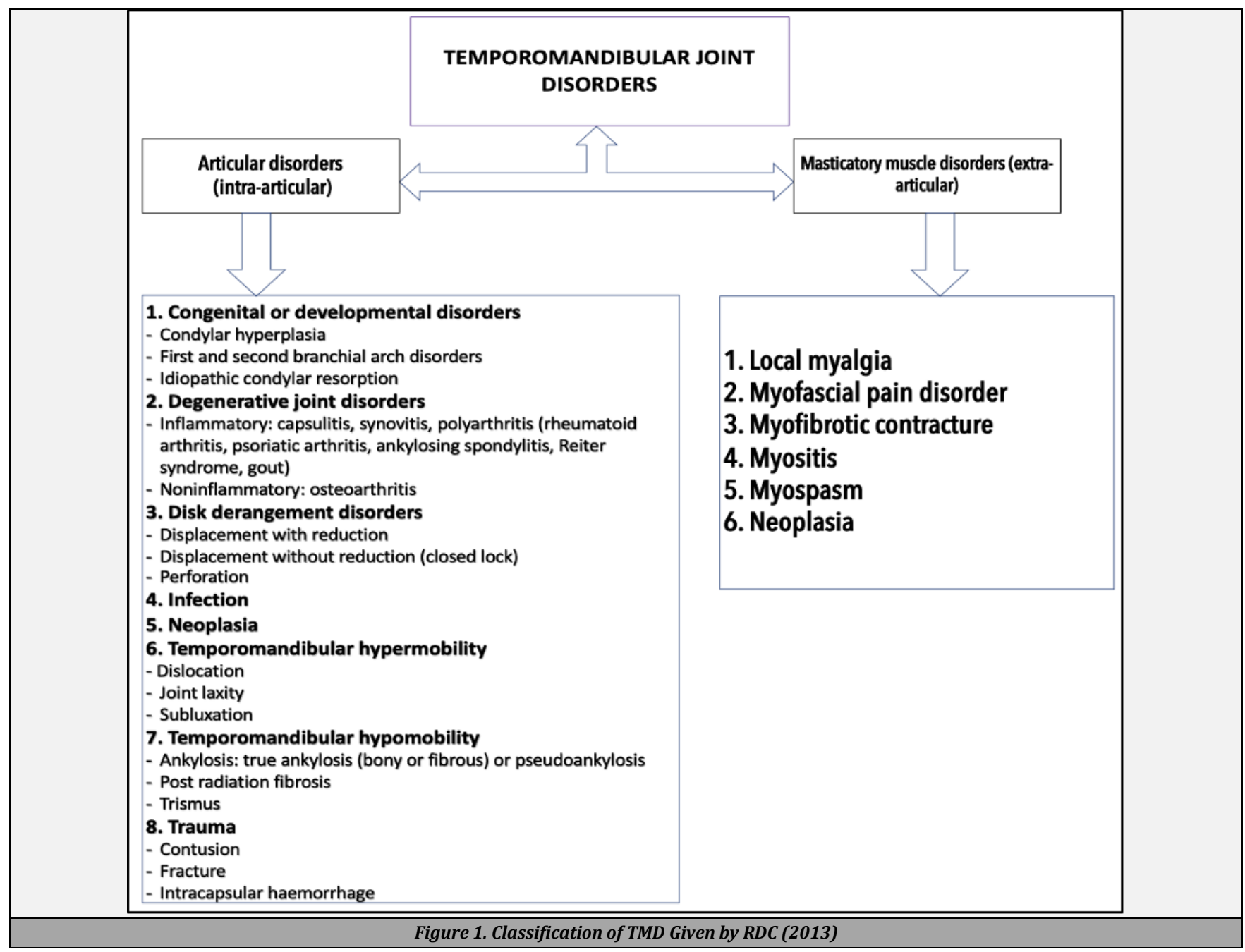




\section{Classification}

TMD is broadly divided into two groups, intra-articular (within the joint) or extra-articular (involving the surrounding musculature). ${ }^{19}$ In 2013, the International Research Diagnostic Criteria for Temporomandibular Dysfunction Consortium Network published an updated classification structure for TMD. 20

\section{DIAGNOSIS OF TMD}

Proper history, physical and radiographic examination can aid in diagnosing the type and aetiology for TMD. During the history taking it is necessary to question the patients on the duration of pain, pain intensity, frequency of the pain, history of any injury or RTA, habitual history, and associated symptoms. ${ }^{7}$ Questioning the patients on their birth history can provide an idea if the patients were delivered via obstetrical forceps. It has been reported in the past that forceps delivery can affect facial growth and can cause fractures in the orofacial region. The fractures were reported to be involving the internal and external auditory canal and may pass through the temporomandibular joint and the mastoid or sigmoid plates. ${ }^{21}$ Analysis of the patient's physical and behavioural status is necessary to rule out syndromes. Hypermobility of the condyle is believed to be linked with connective tissue disorders such as Marfan syndrome or conditions like Downs syndrome.

Signs and symptoms of TMD often include clicking noise (grating, popping, crepitus) which can be present unilaterally and bilaterally during mouth opening or closing, pain during mouth opening and closing, yawning and during mastication, deviation of the jaw, locking of the jaw and muscle tenderness are the frequently occurring ones. ${ }^{22}$ Restricted jaw movements and mouth opening are also to be evaluated during examination. Cases have been reported in the past with the patients reporting only clicking or grating noise without any pain. Examination of crepitus is often done with a stethoscope which is placed on the TMJ region externally.

Pain can also be analysed by being radiated to the surrounding structures on the affected side. Cooper et al. ${ }^{23}$ carried out a large population-based study for 25 years among 4528 people and concluded in his study that the most common symptoms were facial pain (96\%), ear discomfort (82\%), headache (79\%), and jaw discomfort / dysfunction (75\%). Other conditions were mimicking temporomandibular joint or myofascial pain dysfunction syndrome, namely, Giant cell arteritis which has been seen predominantly in the temporal region. Migraine headache is also reported to be involving the temporal region on the affected side; however, it is necessary to rule out the other symptoms which can differentiate from TMJ related pain. Neuropathic pains, such as trigeminal neuralgia, which is a unilateral condition, are also sometimes confused with TMJ pain. Masseter, temporalis, and surrounding muscle tenderness can distinguish myalgia, myofascial trigger zones, or referred pain.

Radiographic imaging such as orthopantomogram (OPG), TMJ tomography, computed tomography (CT) and magnetic resonance imaging (MRI) are extensively used for the diagnosis of TMDs. ${ }^{24}$ Usage of conventional two-dimensional radiographic techniques such as transcranial, trans pharyngeal and transorbital have been reduced due to advancements in three-dimensional imaging modalities. However, the first line of radiographic investigations to be performed is a two-dimensional radiograph. Fractures related to TMJ present acutely, dislocations, and degenerative diseases are often visible in these radiographs. Disc pathologies, fluid effusion to name a few are best seen in MRI. It is also necessary to keep in mind that there is a 78 - $95 \%$ correlation between MRI findings and joint morphology in symptomatic patients, possibilities of false - positive findings occur in $20 \%$ to $34 \%$ of asymptomatic patients. Arthritides and inflammatory conditions can also be seen in MRI. Multi slice CT scans are particularly advised for conditions like fractures, bony pathologies, and neoplasia. Ultrasonography (US) is employed in recent times if MRI is unavailable. It is low in cost and non-invasive. US is mainly used in evaluating the internal derangement of the TMJ. ${ }^{25-29}$

Diagnostic injections consisting of local anaesthesia administered at the auriculotemporal nerve region can be used to differentiate the pain arising from TMD from other orofacial pain. As this procedure is technique sensitive, it is advised to be administered carefully under observation to reduce the complication rate. Other injections such as corticosteroid injections are also used in the TMJ pain, however, these are mainly a treatment modality and are used to reduce the underlying TMJ inflammation.

\section{MANAGEMENT OF TMD}

Planning of the TMD treatment should be started mandatorily as soon as the cause of the TMD is identified. A multidisciplinary approach can be a successful method for the management of TMD with the initial treatment goals focusing mainly on resolving the pain and dysfunction. Various treatment modalities are available in the recent times. Medical management with systemic and topical drug therapy, physical therapy, prosthetic rehabilitation, orthodontic corrections, cognitive behavioural management, and surgical managements are the different treatment options for TMD. Cognitive behavioural therapy can be an effective long term symptomatic treatment for TMD. Proper education related to stress, sleep disorders and parafunctional habits should be explained to the patients. Behavioural changes and lifestyle modifications have proven to be promising in the longer run.

Nonsteroidal anti-inflammatory drugs (NSAIDs) are the first line of drugs administered to patients for a duration of one - two weeks initially as they have proven to reduce both pain and any associated inflammation. ${ }^{31-33}$ Recent evidence-based literature explained that anticonvulsants, NSAIDs, muscle relaxants, tricyclic antidepressants, benzodiazepines and hyaluronate are being used as an effective pain reliever in the management of TMD. ${ }^{32,34-40}$ In an online TMD registry, it was reported that more than 1,500 persons had received antiinflammatory agents (73\%), over the counter pain relievers (56\%), antidepressants (50\%), opioids (48\%), anxiolytics (41\%), and muscle relaxants $(40 \%)^{30}$ due to pain. Administration of corticosteroids (triamcinolone acetonate, methylprednisolone) in the form of intra-articular injection or systemic tablets have been studied previously. 


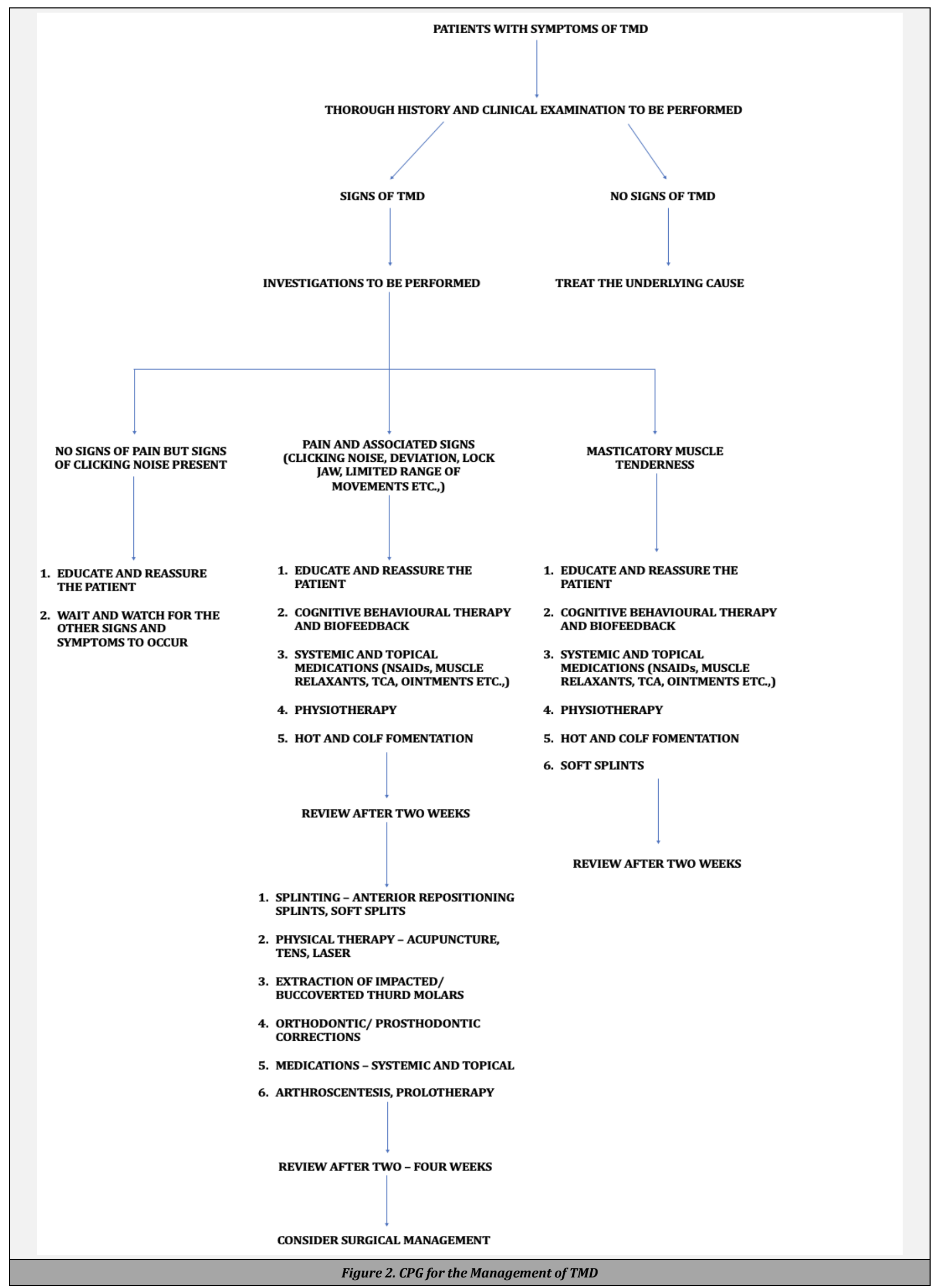

The results of the study advised to restrict the usage of these medications only in severe inflammatory joint conditions associated with underlying autoimmune disorder as reports of articular cartilage damage were present in those 
patients. Anticonvulsants and benzodiazepines are proven to be significantly effective in reducing the pain. They also help in TMD patients with sleep improvement.

Tricyclic antidepressants (amitriptyline) administered up to $15 \mathrm{mg}$ for a period of 10 - 14 days showed a drastic relief in pain, muscle tenderness and improvement in sleep in patients with stress associated TMD at our institution explains that tricyclic antidepressants are also an effective treatment modality.

A survey published revealed that $65 \%$ of TMD patients used hot and cold fomentation to relieve the pain. It was reported that $74 \%$ of patients with TMD were in favour of using hot and cold fomentation as a home remedy to relieve the pain. The science behind advising them is that heat helps in relaxing muscles and increases the blood flow and cold fomentation like ice is used in reducing the swelling if present, and pain. ${ }^{41}$ In our institutional practice, when patients were advised to take soft diet, advised to avoid chewing on the symptomatic side and not sleeping on the affected side apart from hot and cold fomentation have proven to show improvement in the symptoms in acute TMD conditions.

The use of splints is thought to diminish the degenerative forces placed on the TMJ, articular disk, and dentition. ${ }^{42}$ Few systematic reviews conducted have shown conflicting results on the preferred splints for relieving TMD symptoms. ${ }^{43}$ However patients with chronic nocturnal bruxism were reported to be benefitted with splints. Similar to the occlusal splints, the anterior repositioning splints are proven to be effective in patients diagnosed with anterior disc displacement. The drawback of these splints is that they cannot be advised for a longer duration.

DeAngelis AF et al. conducted a study among 60 patients who were referred for third molar extraction. Of the 60 patients, they found that $13.3 \%$ showed signs and symptoms of TMJ pain and dysfunction due to third molars. ${ }^{44} \mathrm{~A}$ similar study was conducted in 189 Iraqi population with bilateral maxillary and mandibular impacted third molars to rule out if TMD was caused due to impacted third molars. The author concluded that there was no statistically significant evidence to prove that impacted third molars were an effective factor for causing TMJ dysfunction and pain. ${ }^{45}$ However, based on our personal experiences at our institution, patients with TMD pain and restricted mouth opening were present with impacted and buccoverted third molars. These patients when subjected to extraction showed significant relief in pain and dysfunction post procedure.

Physical therapies such as TENS, acupuncture have been used frequently and proven to be effective in treating TMD associated with myofascial pain. ${ }^{46}$ TENS is a physical therapy which can be used by placing the electrodes on the affected area in the orofacial region and can be given up to 45 mins depending upon the frequency (high or low) and patient tolerance. Acupuncture can be administered for about 30 mins during each session and the average period was 4 weeks. The main disadvantage of TENS is it is contraindicated in patients with prosthesis or implants anywhere in the body, patients with cardiac pacemakers etc. However, there is no significantly evident data to support this theory. They are also contraindicated in patients with facial lesions.

Arthrocentesis or proliferation therapy (prolotherapy) are said to be the new hopes in managing the TMD pain. They have been reported to be effective in stabilising the injured temporomandibular joint and in relieving the joint pain.
However, studies have proven to advise to stop NSAIDs 2 - 3 days prior and up to 2 weeks for prolotherapy. 47

Surgical management of TMDs are predominantly seen in cases of trauma or injury to the TMJ. The literature states that 5 - 10 percent of all patients undergoing treatment for TMDs require surgical intervention. ${ }^{16,17,48-50}$ Modified condylotomy, open joint surgery, joint replacement and arthroscopy are a few invasive procedures carried out in TMJ. It is necessary to advise the patients for a full recovery which is about $2-6$ weeks in duration. Post procedural care is a must for these patients, in case of rushing might cause furthermore damage to the jaw.

\section{CONCLUSIONS}

To conclude, the decision on which treatment modality is best suited for which type of TMD is completely based on a physician's thorough knowledge on the subject and the presenting confounding factors of the patient with TMD.

Financial or other competing interests: None.

Disclosure forms provided by the authors are available with the full text of this article at jemds.com.

\section{REFERENCES}

[1] Lim PF, Smith S, Bhalang K, et al. Development of temporomandibular disorders is associated with greater bodily pain experience. Clin J Pain 2010;26(2):116-20.

[2] Gonçalves DAG, Camparis CM, Speciali JG, et al. Temporomandibular disorders are differentially associated with headache diagnoses: a controlled study. Clin J Pain 2011;27(7):611-5.

[3] Maixner W, Diatchenko L, Dubner R, et al. Orofacial pain prospective evaluation and risk assessment study--the OPPERA study. J Pain 2011;12(11 Suppl):T4-11.e1-2.

[4] Kim KW, Ha IH, Lee YJ, et al. A clinical practice guideline for temporomandibular disorders in traditional Korean medicine: An evidence-based approach. Eur J Integr Med 2018;23:123-33.

[5] De Leeuw R. American academy of orofacial pain: guidelines for assessment, diagnosis and management. Quintessence Publishing Company, Incorporated 2018: p. 327.

[6] Yost O, Liverman CT, English R, et al. Temporomandibular disorders: priorities for research and care. Washington: National Academies Press 2020: p. 426.

[7] Gauer RL, Semidey MJ. Diagnosis and treatment of temporomandibular disorders. Am Fam Physician 2015;91(6):378-86.

[8] Clinical Affairs Committee-Temporomandibular Joint Problems in Children Subcommittee, American Academy of Pediatric Dentistry. Guideline on acquired temporomandibular disorders in infants, children, and adolescents. Pediatr Dent 2015;37(5):78-84.

[9] American Society of Temporomandibular Joint Surgeons. Guidelines for diagnosis and management of disorders involving the temporomandibular joint and related musculoskeletal structures. Cranio 2003;21(1):68-76.

[10] Sanders AE, Maixner W, Nackley AG, et al. Excess risk of 
temporomandibular disorder associated with cigarette smoking in young adults. J Pain 2012;13(1):21-31.

[11] Roldán-Barraza C, Janko S, Villanueva J, et al. A systematic review and meta-analysis of usual treatment versus psychosocial interventions in the treatment of myofascial temporomandibular disorder pain. J Oral Facial Pain Headache 2014;28(3):205-22.

[12] Fricton J. Current evidence providing clarity in management of temporomandibular disorders: summary of a systematic review of randomized clinical trials for intra-oral appliances and occlusal therapies. J Evid Based Dent Pract 2006;6(1):48-52.

[13] Butts R, Dunning J, Pavkovich R, et al. Conservative management of temporomandibular dysfunction: a literature review with implications for clinical practice guidelines (Narrative review part 2). J Bodyw Mov Ther 2017;21(3):541-8.

[14] De Paula Gomes CAF, Politti F, Andrade DV, et al. Effects of massage therapy and occlusal splint therapy on mandibular range of motion in individuals with temporomandibular disorder: a randomized clinical trial. J Manipulative Physiol Ther 2014;37(3):164-9.

[15] Garrigós-Pedrón M, La Touche R, Navarro-Desentre P, et al. Effects of a physical therapy protocol in patients with chronic migraine and temporomandibular disorders: a randomized, single-blinded, clinical trial. J Oral Facial Pain Headache 2018;32(2):137-50.

[16] Dimitroulis G. Temporomandibular joint surgery: what does it mean to the dental practitioner? Aust Dent J 2011;56(3):257-64.

[17] Reston JT, Turkelson CM. Meta-analysis of surgical treatments for temporomandibular articular disorders. J Oral Maxillofac Surg 2003;61(1):3-12.

[18] Dimitroulis G. Management of temporomandibular joint disorders: a surgeon's perspective. Aust Dent J 2018;63(Suppl 1):S79-90.

[19] Okeson JP. Joint intracapsular disorders: diagnostic and nonsurgical management considerations. Dent Clin North Am 2007;51(1):85-103, vi.

[20] Schiffman E, Ohrbach R, Truelove E, et al. Diagnostic Criteria for Temporomandibular Disorders (DC/TMD) for clinical and research applications: recommendations of the international RDC/TMD Consortium network* and orofacial pain special interest group. J Oral Facial Pain Headache 2014;28(1):6-27.

[21] Germane N, Rubenstein L. The effects of forceps delivery on facial growth. Pediatr Dent 1989;11(3):193-7.

[22] Scrivani SJ, Keith DA, Kaban LB. Temporomandibular disorders. N Engl J Med 2008;359(25):2693-705.

[23] Cooper BC, Kleinberg I. Examination of a large patient population for the presence of symptoms and signs of temporomandibular disorders. Cranio 2007;25(2):11426.

[24] Hunter A, Kalathingal S. Diagnostic imaging for temporomandibular disorders and orofacial pain. Dent Clin North Am 2013;57(3):405-18.

[25] Emshoff R, Innerhofer K, Rudisch A, et al. Clinical versus magnetic resonance imaging findings with internal derangement of the temporomandibular joint: an evaluation of anterior disc displacement without reduction. J Oral Maxillofac Surg 2002;60(1):36-41.

[26] Bertram S, Rudisch A, Innerhofer K, et al. Diagnosing TMJ internal derangement and osteoarthritis with magnetic resonance imaging. J Am Dent Assoc 2001;132(6):753-61.

[27] Maizlin ZV, Nutiu N, Dent PB, et al. Displacement of the temporomandibular joint disk: correlation between clinical findings and MRI characteristics. J Can Dent Assoc 2010;76:a3.

[28] Kircos LT, Ortendahl DA, Mark AS, et al. Magnetic resonance imaging of the TMJ disc in asymptomatic volunteers. J Oral Maxillofac Surg 1987;45(10):852-4.

[29] Lamot U, Strojan P, Popovič KS. Magnetic resonance imaging of temporomandibular joint dysfunctioncorrelation with clinical symptoms, age and gender. Oral Surg Oral Med Oral Pathol Oral Radiol 2013;116(2):25863.

[30] Hoffmann RG, Kotchen JM, Kotchen TA, et al. Temporomandibular disorders and associated clinical comorbidities. Clin J Pain 2011;27(3):268-74.

[31] Hersh EV, Balasubramaniam R, Pinto A. Pharmacologic management of temporomandibular disorders. Oral Maxillofac Surg Clin North Am 2008;20(2):197-210, vi.

[32] Ta LE, Dionne RA. Treatment of painful temporomandibular joints with a cyclooxygenase-2 inhibitor: a randomized placebo-controlled comparison of celecoxib to naproxen. Pain 2004;111(1-2):13-21.

[33] List T, Axelsson S, Leijon G. Pharmacologic interventions in the treatment of temporomandibular disorders, atypical facial pain and burning mouth syndrome. A qualitative systematic review. Journal of Prosthetic Dentistry 2004;91(5):500.

[34] Kimos P, Biggs C, Mah J, et al. Analgesic action of gabapentin on chronic pain in the masticatory muscles: a randomized controlled trial. Pain 2007;127(1-2):151-60.

[35] Martin WJJM, Perez RSGM, Tuinzing DB, et al. Efficacy of antidepressants on orofacial pain: a systematic review. Int J Oral Maxillofac Surg 2012;41(12):1532-9.

[36] Singer E, Sharav Y, Dubner R, et al. The efficacy of diazepam and ibuprofen in the treatment of chronic myofascial orofacial pain. Pain 1987;30:S83.

[37] Denucci DJ, Sobiski C, Dionne RA. Triazolam improves sleep but fails to alter pain in TMD patients. J Orofac Pain 1998;12(2):116-23.

[38] Rizzatti-Barbosa CM, Nogueira MTP, De Andrade ED, et al. Clinical evaluation of amitriptyline for the control of chronic pain caused by temporomandibular joint disorders. Cranio 2003;21(3):221-5.

[39] Ekberg EC, Kopp S, Akerman S. Diclofenac sodium as an alternative treatment of temporomandibular joint pain. Acta Odontol Scand 1996;54(3):154-9.

[40] Jagger RG. Diazepam in the treatment of temporomandibular joint dysfunction syndrome--a double blind study. J Dent 1973;2(1):37-40.

[41] Furlan RMMM, Giovanardi RS, De Oliveira E Britto ATB, et al. The use of superficial heat for treatment of temporomandibular disorders: an integrative review. Codas 2015;27(2):207-12.

[42] Klasser GD, Greene CS. Oral appliances in the management of temporomandibular disorders. Oral Surg Oral Med Oral Pathol Oral Radiol Endod 2009;107(2):212-23.

[43] Al-Ani MZ, Davies SJ, Gray RJM, et al. Stabilisation splint therapy for temporomandibular pain dysfunction syndrome. Cochrane Database Syst Rev 2004;1:CD002778.

[44] Deangelis AF, Chambers IG, Hall GM. Temporomandibular 
joint disorders in patients referred for third molar extraction. Aust Dent J 2009;54(4):323-5.

[45] Jasim H. Effect of the impacted third molars on the development of temporomandibular joint (TMJ) clicking. Journal of International Oral Health 2019;11(6):393-7.

[46] Rosted P. Practical recommendations for the use of acupuncture in the treatment of temporomandibular disorders based on the outcome of published controlled studies. Oral Dis 2001;7(2):109-15.

[47] Kumar A, Naik P, Jaishankar HP, et al. Prolotherapy: a new hope for temporomandibular joint pain. Indian J Pain
2013;27(2):49-52.

[48] Dimitroulis G. Temporomandibular disorders: a clinical update. BMJ 1998;317(7152):190-4.

[49] Dolwick MF, Dimitroulis G. Is there a role for temporomandibular joint surgery? Br J Oral Maxillofac Surg 1994;32(5):307-13.

[50] Dimitroulis G. The role of surgery in the management of disorders of the temporomandibular joint: a critical review of the literature Part I. Int J Oral Maxillofac Surg 2005;34(2):107-13. 